SECTION 1. Theoretical research in mathematics.

Galiaskarova Guzeliya Rafkatovna

BASHKIR STATE UNIVERSITY Sterlitamak branch candidat of physical and mathematical sciences, lecturer

\title{
A RELATION OF THE DARBOUX PROBLEM FOR THE TELEGRAPH EQUATION WITH A DEPARTURE FROM THE CHARACTERISTICS
}

The article deals with the problem of constructing solutions of the Darboux problem for the case of the Riemann method departing from the characteristics. A function of the RiemannHadamard for the specified region. Using the Riemann-Hadamard constructed a solution of the Darboux problem and obtained a relation on the characteristic equation.

Keywords: Darboux problem, the Riemann-Hadamard function, telegraph equation

\section{ОБ ОДНОМ СООТНОШЕНИИ ЗАДАЧИ ДАРБУ ДЛЯ ТЕЛЕГРАФНОГО УРАВНЕНИЯ С ОТХОДОМ ОТ ХАРАКТЕРИСТИКИ}

В статье рассматривается проблема построения решения задачи Дарбу методом Римана для случая отхода от характеристики. Построена функция РиманаАдамара для заданной области. Используя функцию Римана-Адамара, построено решение задачи Дарбу и получено соотношение на характеристике уравнения.

Ключевые слова: задача Дарбу, функиия Римана-Адамара, телеграфное уравнение

Рассмотрим уравнение

$$
L u=u_{\xi \eta}+c u=0,
$$

где $c$ - произвольное действительное число, в области $\Delta=\{(\xi, \eta) \mid 0<\xi<\eta<\alpha \xi<1, \alpha>1\}$

Для данного уравнения поставим задачу Дарбу:

Задача $D^{\prime}$. Найти в области $\Delta$ функцию $u(\xi, \eta)$, удовлетворяющую условиям

$$
\begin{aligned}
& u(\xi, \eta) \in C(\bar{\Delta}) \wedge C^{1}(\Delta \cup\{\eta=\alpha \xi\}), u_{\xi \eta} \in C(\Delta) ; L u(\xi, \eta) \equiv 0,(\xi, \eta) \in \Delta, \\
& \left.u(\xi, \eta)\right|_{\eta=\alpha \xi}=\tau(\xi), 0 \leq \xi \leq \frac{1}{\alpha} ; u(\xi, \eta)_{\eta=\xi}=\psi(\xi), 0 \leq \xi \leq 1 ; \psi(0)=\varphi(0) .
\end{aligned}
$$

Для решения задачи $D^{\prime}$ применим метод Римана-Адамара, который основан на так называемой функции Римана-Адамара. Ранее этот метод применялся в работах [1, c.73], [4, с.67],[3, с.71]. В частности, в работе [2, с.1] была построена функция РиманаАдамара для области, ограниченной прямыми $\eta=\xi, \xi=0, \eta=1$. В представленной статье характеристика уравнения $\xi=0$ заменена прямой $\eta=\alpha \xi$, что значительно усложняет процесс построения функции.

Разобьем область $\Delta$ на следующие подобласти (в скобках указаны прямые, которые ограничивают указанные области:

$$
\sigma_{2 k}:\left\{\eta=\alpha \xi, \xi=\frac{\eta_{0}}{\alpha^{k+1}}, \eta=\frac{\xi_{0}}{\alpha^{k}}\right\}, \sigma_{2 k+1}:\left\{\eta=\alpha \xi, \xi=\frac{\eta_{0}}{\alpha^{k+1}}, \eta=\frac{\xi_{0}}{\alpha^{k+1}}\right\}
$$




$$
\begin{gathered}
\omega_{0}=\left\{\eta=\eta_{0}, \xi=\xi_{0}, \eta=\xi_{0}, \xi=\frac{\eta_{0}}{\alpha}\right\} \omega_{2 k}:\left\{\xi=\frac{\eta_{0}}{\alpha^{k+1}} \xi=\frac{\xi_{0}}{\alpha^{k}}, \eta=\frac{\eta_{0}}{\alpha^{k}}, \eta=\frac{\xi_{0}}{\alpha^{k}}\right\}, \\
\omega_{2 k+1}:\left\{\xi=\frac{\eta_{0}}{\alpha^{k+1}} \xi=\frac{\xi_{0}}{\alpha^{k+1}}, \eta=\frac{\eta_{0}}{\alpha^{k+1}}, \eta=\frac{\xi_{0}}{\alpha^{k}}\right\}, \Delta_{2 k+1}:\left\{\eta=\xi, \xi=\frac{\eta_{0}}{\alpha^{k+1}}, \eta=\frac{\xi_{0}}{\alpha^{k}}\right\} \\
\Delta_{2 k}:\left\{\eta=\xi, \xi=\frac{\xi_{0}}{\alpha^{k}}, \eta=\frac{\eta_{0}}{\alpha^{k}}\right\} .
\end{gathered}
$$

Построим в области $\Delta$ функцию Римана-Адамара, задав её в каждой из указанных областей, следующим образом

1. $R\left(\xi, \eta, \xi_{0}, \eta_{0}\right)$, как функция от $(\xi, \eta)$ является решением сопряженного уравнения $L^{*} u=0$, которое в данном случае является самосопряженным и, значит, совпадает с уравнением $L u=0$.

2. $R_{\xi}=0$ на $\eta=\eta_{0} ; R_{\eta}=0$ на $\xi=\xi_{0} ; R=0$ на $\eta=\xi$ и $\eta=\alpha \xi$.

3. $\frac{\partial\left[R_{1}\right]}{\partial \xi}=0$, где $\left[R_{1}\right]=\lim _{\varepsilon \rightarrow 0}\left(R\left(\xi ; \frac{\xi_{0}}{\alpha^{k}}+\varepsilon ; \xi_{0} ; \eta_{0}\right)-R\left(\xi ; \frac{\xi_{0}}{\alpha^{k}}-\varepsilon ; \xi_{0} ; \eta_{0}\right)\right)$

$\frac{\partial\left[R_{2}\right]}{\partial \xi}=0$, где $\left[R_{2}\right]=\lim _{\varepsilon \rightarrow 0}\left(R\left(\xi ; \frac{\eta_{0}}{\alpha^{k}}+\varepsilon ; \xi_{0} ; \eta_{0}\right)-R\left(\xi ; \frac{\eta_{0}}{\alpha^{k}}-\varepsilon ; \xi_{0} ; \eta_{0}\right)\right)$

$\frac{\partial\left[R_{3}\right]}{\partial \eta}=0$, где $\left[R_{3}\right]=\lim _{\varepsilon \rightarrow 0}\left(R\left(\frac{\xi_{0}}{\alpha^{k}}+\varepsilon ; \eta ; \xi_{0} ; \eta_{0}\right)-R\left(\frac{\xi_{0}}{\alpha^{k}}-\varepsilon ; \eta ; \xi_{0} ; \eta_{0}\right)\right)$

$\frac{\partial\left[R_{4}\right]}{\partial \xi}=0$, где $\left[R_{4}\right]=\lim _{\varepsilon \rightarrow 0}\left(R\left(\frac{\eta_{0}}{\alpha^{k}}+\varepsilon ; \xi ; \xi_{0} ; \eta_{0}\right)-R\left(\frac{\eta_{0}}{\alpha^{k}}-\varepsilon ; \xi ; \xi_{0} ; \eta_{0}\right)\right)$

4. $R\left(\xi, \eta, \xi_{0}, \eta_{0}\right)=1$

Функция Римана-Адамара задачи $D^{\prime}$ определяется следующим образом

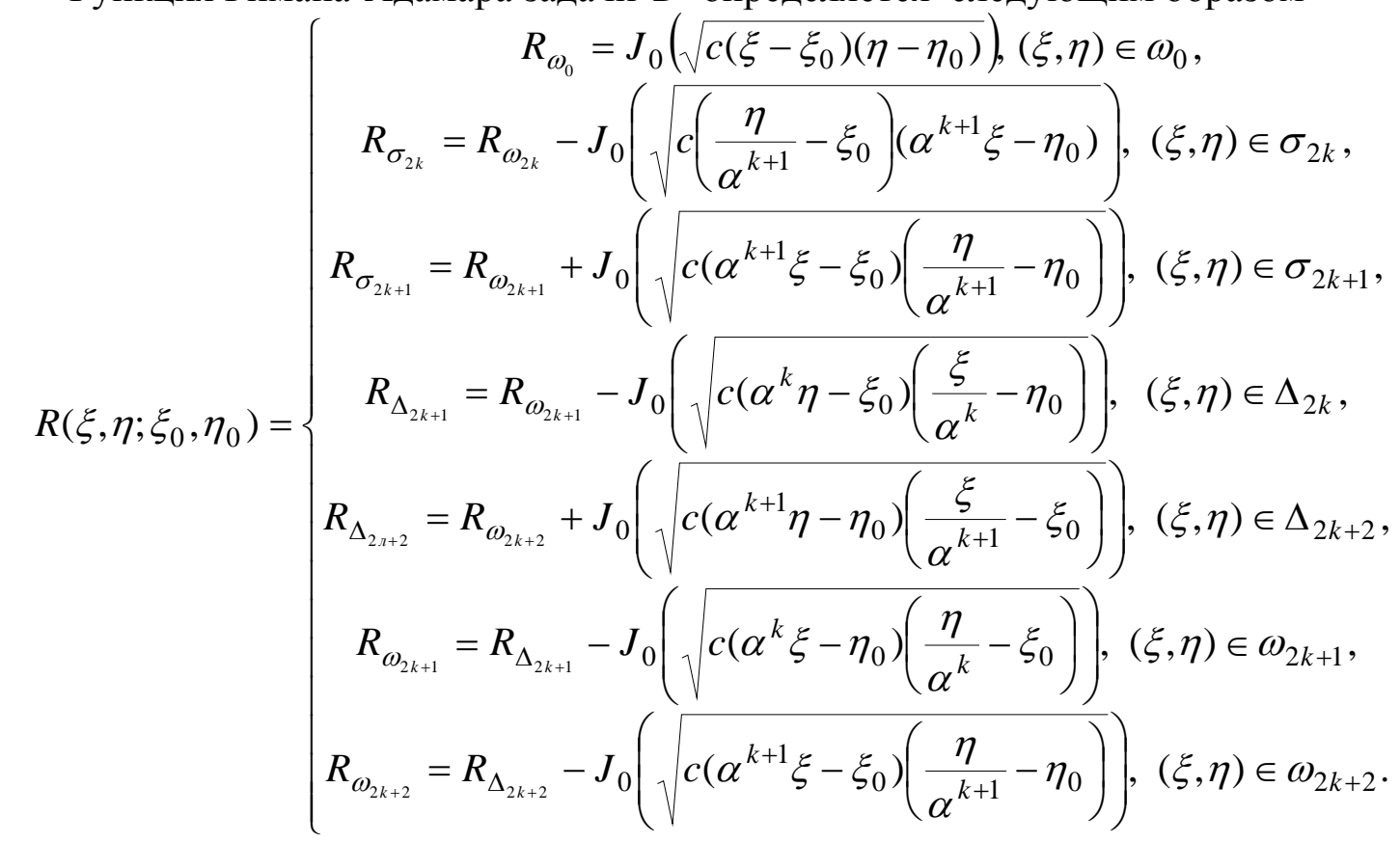

где $J_{0}(\cdot)$ - функция Бесселя нулевого порядка.

Запишем тождество Грина для оператора $L$ :

$$
u \cdot L R-R \cdot L u=\frac{1}{2}\left(u R_{\eta}-R u_{\eta}\right)_{\xi}+\frac{1}{2}\left(u R_{\xi}-R u_{\xi}\right)_{\eta}
$$


Выполняя стандартную процедуру, состоящую в интегрировании тождества Грина по области $\Delta_{1} \cup \Delta_{2}$ и применении формулы Гаусса-Остроградского, получим

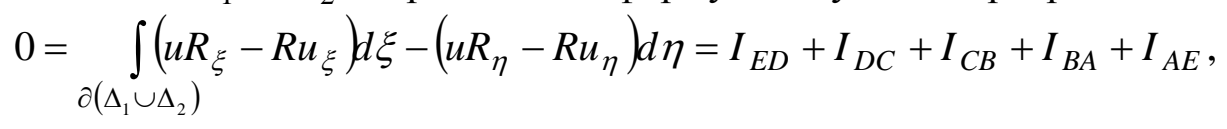

где $D=\left(\xi_{0}, \eta_{0}\right), C=\left(\frac{\eta_{0}}{\alpha}, \eta_{0}\right), B=\left(\frac{\xi_{0}}{\alpha}, \xi_{0}\right), A=(0,0), E=\left(\xi_{0}, \xi_{0}\right)$.

Вычислим интегралы $I_{E D}, I_{D C}, I_{C B}, I_{B A}, I_{A E}$ :

$$
\begin{aligned}
& I_{E D}=\left.u R_{1}\right|_{E} ^{D}=u R_{1}(D)-u R_{1}(E)=u\left(\xi_{0}, \eta_{0}\right)-\tau\left(\xi_{0}\right) \\
& I_{D C}=-\left.u R_{1}\right|_{D} ^{C}=-u R_{1}(C)+u R_{1}(D)=-\psi\left(\frac{\eta_{0}}{\alpha}\right)+u\left(\xi_{0}, \eta_{0}\right) \\
& I_{A C}=\sum_{n=1}^{\infty}\left(\int_{0}^{\frac{\eta_{0}}{\alpha^{n}}} u\left(R_{\xi} d \xi-R_{\eta} d \eta\right)+\left.\int_{0}^{\frac{\xi_{0}}{\alpha^{n}}} u\left(R_{\xi} d \xi-R_{\eta} d \eta\right)\right|_{\eta=\alpha \xi}=\right. \\
& =\sum_{n=1}^{\infty} \frac{c\left(\eta_{0}-\alpha^{2 n+1} \xi_{0}\right)}{2} \int_{0}^{\frac{\xi_{0}}{\alpha^{n}}} \frac{J_{1}\left(\sqrt{c\left(\alpha^{n+1} \xi-\xi_{0}\right)\left(\frac{\xi}{\alpha^{n}}-\eta_{0}\right)}\right)}{\sqrt{\left(\alpha^{n} \xi-\alpha^{2 n} \xi_{0}\right)\left(\alpha^{n+1} \xi-\eta_{0}\right)} \tau(\xi) d \xi+}
\end{aligned}
$$

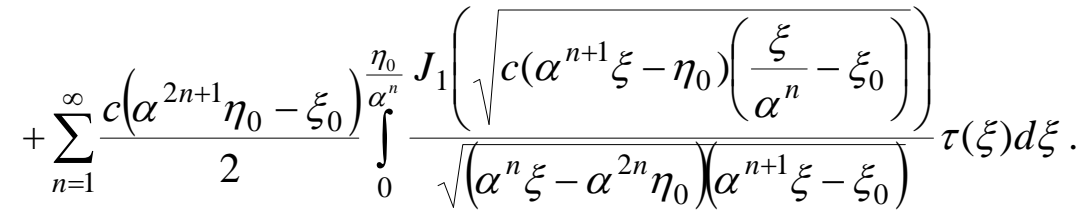

$$
\begin{aligned}
& I_{A E}=\sum_{n=1}^{\infty}\left(\int_{0}^{\frac{\eta_{0}}{\alpha^{n}}} u\left(R_{\xi} d \xi-R_{\eta} d \eta\right)+\left.\int_{0}^{\frac{\xi_{0}}{\alpha^{n}}} u\left(R_{\xi} d \xi-R_{\eta} d \eta\right)\right|_{\eta=\xi}=\right.
\end{aligned}
$$

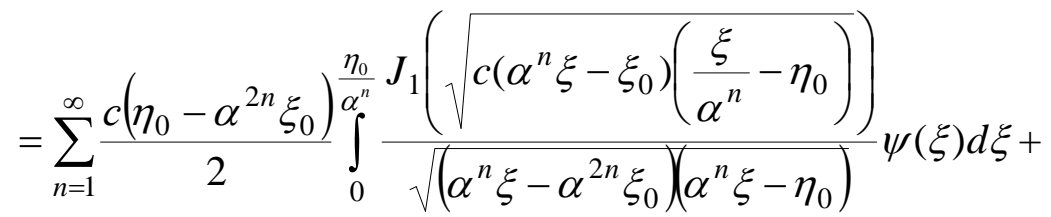

$$
\begin{aligned}
& +\sum_{n=1}^{\infty} \frac{c\left(\alpha^{2 n+1} \eta_{0}-\xi_{0}\right)}{2} \int_{0}^{\frac{\eta_{0}}{\alpha^{n}}} \frac{J_{1}\left(\sqrt{c\left(\alpha^{n+1} \xi-\xi_{0}\right)\left(\frac{\xi}{\alpha^{n}}-\eta_{0}\right)}\right)}{\sqrt{\left(\alpha^{n} \xi-\alpha^{2 n} \xi_{0}\right)\left(\alpha^{n+1} \xi-\eta_{0}\right)}} \psi(\xi) d \xi .
\end{aligned}
$$

Подставляя, полученные интегралы в формулу и, проводя дополнительные преобразования, окончательно получим.

$$
\begin{gathered}
u(\xi, \eta)=\tau(\xi)+\psi\left(\frac{\eta}{\alpha}\right)-\frac{c(\eta-\xi)}{4} \int_{0}^{\xi} \frac{J_{1}(\sqrt{c(t-\xi)(t-\eta)})}{\sqrt{(t-\xi)(t-\eta)}} \tau(t) d t+ \\
+\sum_{n=1}^{\infty} \frac{c\left(\eta-\alpha^{2 n+1} \xi\right)}{4} \int_{0}^{\frac{\xi}{\alpha^{n}}} \frac{J_{1}\left(\sqrt{\left.c\left(\alpha^{n+1} t-\xi\right)\left(\frac{t}{\alpha^{n}}-\eta\right)\right)}\right.}{\sqrt{\left(\alpha^{n} t-\alpha^{2 n} \xi\right)\left(\alpha^{n+1} t-\eta\right)} \tau(t) d t+}
\end{gathered}
$$




$$
\begin{aligned}
& +\sum_{n=1}^{\infty} \frac{c\left(\alpha^{2 n+1} \eta-\xi\right)}{4} \int_{0}^{\frac{\eta}{\alpha^{n}}} \frac{J_{1}\left(\sqrt{ }\left(\alpha^{n+1} t-\eta\right)\left(\frac{t}{\alpha^{n}}-\xi\right)\right)}{\sqrt{\left(\alpha^{n} t-\alpha^{2 n} \eta\right)\left(\alpha^{n+1} t-\xi\right)} \tau(t) d t+} \\
& +\sum_{n=1}^{\infty} \frac{c\left(\eta-\alpha^{2 n} \xi\right)}{4} \int_{0}^{\frac{\xi}{\alpha^{n}}} \frac{J_{1}\left(\sqrt{ }\left(\alpha^{n} t-\xi\right)\left(\frac{t}{\alpha^{n}}-\eta\right)\right)}{\sqrt{\left(\alpha^{n} t-\alpha^{2 n} \xi\right)\left(\alpha^{n} t-\eta\right)}} \psi(t) d t+ \\
& +\sum_{n=1}^{\infty} \frac{c\left(\alpha^{2 n+1} \eta-\xi\right)}{4} \int_{0}^{\frac{\eta}{\alpha^{n}}} \frac{\left.J_{1}\left(\sqrt{c} \alpha^{n+1} t-\xi\right)\left(\frac{t}{\alpha^{n}}-\eta\right)\right)}{\sqrt{\left(\alpha^{n} t-\alpha^{2 n} \xi\right)\left(\alpha^{n+1} t-\eta\right)} \psi(t) d t .}
\end{aligned}
$$

Теорема. Если функция $\tau(\xi) \in C^{1}\left[0, \frac{1}{\alpha}\right], \alpha>1, a \psi(\xi) \in C^{2}[0,1]$, то существует единственное решение задачи $D^{\prime}$ и оно определяется формулой (2).

Положим в равенстве (2) $\xi=0$, тогда

$$
\begin{aligned}
& u(0, \eta)=\tau(0)+\psi\left(\frac{\eta}{\alpha}\right)+\sum_{n=1}^{\infty} \frac{c \alpha^{2 n+1} \eta}{4} \int_{0}^{\frac{\eta}{\alpha^{n}}} \frac{J_{1}\left(\sqrt{c \frac{t}{\alpha^{n}}\left(\alpha^{n+1} t-\eta\right)}\right)}{\sqrt{\alpha^{n+1} t\left(\alpha^{n} t-\alpha^{2 n} \eta\right)}} \tau(t) d t+
\end{aligned}
$$

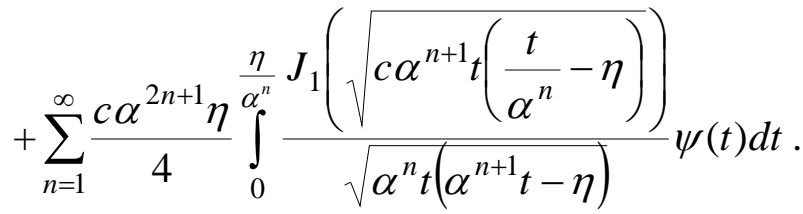

\section{Литература}

1. Сабитов К. Б., Акимов А. А. К теории аналога задачи Неймана для уравнения смешанного типа // Известия ВУЗов.Математика. 2001. № 10. С.73 - 80.

2. Gellerstedt S. Quelques problemes mixtes pour l'equation $y^{m} z_{x x}+z_{y y}=0 / /$ Arkiv for Matematik,Astronomi o ch Fysik. 1937. 26A (3). P. 1 - 32.

3. Акимов А. А. Задача Моравец для обобщенного уравнения Трикоми // Сибирские электронные математические известия. 2006. Т. 3. С. 71 - 82. http: //semr.math.nsc.ru.

4. Акимов А. А. Об одной теореме единственности задачи Моравец // Альманах современной науки и образования. 2010. № 12. С. 67-69. 